\title{
Stability Theorems for Two Measures of Symmetry
}

\author{
H. Groemer \\ Department of Mathematics, The University of Arizona, \\ Tucson, AZ 85721, USA \\ groemer@math.arizona.edu
}

\begin{abstract}
This article concerns two measures of central symmetry that characterize cones as the most asymmetric convex bodies. Stability estimates are established that provide information on the deviation of a convex body from a cone if the corresponding measure of symmetry is close to its maximum value.
\end{abstract}

The survey article [6] of Grünbaum shows convincingly that a large variety of results in the theory of convex sets can be viewed as statements regarding certain measures of symmetry (or asymmetry). We consider here two such measures that attain their minimum if the bodies are centrally symmetric and their maximum if the bodies are cones. Our primary objective is to establish stability estimates concerning the maximum value of these measures. In other words, we seek estimates for the deviation of a convex body from a cone if its measure of symmetry is close to its maximum value.

The following definitions and notations are used consistently. $\mathbf{R}^{n}$ denotes the euclidean $n$-dimensional space, where it is always assumed that $n \geq 2$. We let $o$ denote the origin of $\mathbf{R}^{n}$, and $S^{n-1}$ the $(n-1)$-dimensional unit sphere in $\mathbf{R}^{n}$ (centered at $o$ ). The class of convex bodies in $\mathbf{R}^{n}$, that means the compact convex subsets in $\mathbf{R}^{n}$ with nonempty interior, is denoted by $\mathcal{K}^{n} . \Delta$ denotes the symmetric difference metric on $\mathcal{K}^{n}$. Thus, if $K, L \in \mathcal{K}^{n}$, then $\Delta(K, L)=v(K \cup L)-v(K \cap L)$, where $v$ signifies the volume in $\mathbf{R}^{n}$. Instead of "hyperplane" we simply say "plane." If $B$ is a compact convex subset of a plane in $\mathbf{R}^{n}$ and $p$ is a point in $\mathbf{R}^{n}$, then the convex hull of $B \cup\{p\}$, if it has nonempty interior, is called a cone with base $B$ and apex $p$, and it is denoted by $\mathcal{C}(p, B)$. For any $K \in \mathcal{K}^{n}$ we let $z(K)$ denote the centroid of $K$ and $H_{K}(u)$ the support plane of $K$ in the direction $u$. The distance between $z(K)$ and $H_{K}(u)$, that is, the support function of $K$ with respect to the centroid, is denoted by $h_{K}(u)$. 
As our first measure of symmetry we define

$$
\eta(K)=\sup \left\{h_{K}(u) / h_{K}(-u): u \in S^{n-1}\right\} .
$$

Clearly,

$$
\eta(K) \geq 1
$$

and $\eta(K)=1$ holds exactly if $K$ is centrally symmetric. Minkowski already showed that, for all $K \in \mathcal{K}^{n}$,

$$
\eta(K) \leq n .
$$

Furthermore, it is easy to see that if $K$ is a cone, then $\eta(K)=n$, and that $h_{K}(u) / h_{K}(-u)$ attains the value $n$ if $u$ is an inner normal vector of $K$ at its base. For references and historic comments regarding $\eta(K)$ and related functions see [6].

We now can formulate our first result.

Theorem 1. Let there be given a $K \in \mathcal{K}^{n}$ and an $\varepsilon \geq 0$. If $\eta(K) \geq n-\varepsilon$ there exists a cone $C$ such that

$$
\Delta(K, C) \leq \frac{4}{n+1} v(K) \varepsilon
$$

We add some relevant remarks concerning this theorem.

1. Letting $\varepsilon=n-\eta(K)$, which is admissible because of (2), we obtain from (3) the following statement:

For any $K \in \mathcal{K}^{n}$ there is a cone $C$ such that

$$
\eta(K) \leq n-\frac{n+1}{4 v(K)} \Delta(K, C) .
$$

This is a sharpened version of (2). Combined with the previous remark concerning the occurrence of the equality $\eta(K)=n$ one infers that this equality holds if and only if $K$ is a cone.

2. For the inequality (1), where equality holds exactly if $K$ is centrally symmetric, one can also establish a corresponding stability result. To see this, assume that, for some $\varepsilon \geq 0$,

$$
\eta(K) \leq 1+\varepsilon
$$

Then $1 /(1+\varepsilon) \leq h_{K}(u) / h_{K}(-u) \leq 1+\varepsilon$ (for all $\left.u \in S^{n-1}\right)$ and therefore

$$
\left|h_{K}(u)-\frac{1}{2}\left(h_{K}(u)+h_{K}(-u)\right)\right|=\frac{1}{2}\left|\frac{h_{K}(u)}{h_{K}(-u)}-1\right|\left|h_{K}(-u)\right| \leq \frac{n D}{2(n+1)} \varepsilon,
$$

where $D$ denotes the diameter of $K$. Thus, letting $K^{*}$ denote the convex body obtained from $K$ by central symmetrization (with respect to $z(K)$ ), and $\delta$ the Hausdorff metric on $\mathcal{K}^{n}$ we have justified the following statement: 
If $\eta(K) \leq 1+\varepsilon$ there exist a centrally symmetric convex body $K^{*}$ such that

$$
\delta\left(K, K^{*}\right) \leq \frac{n D}{2(n+1)} \varepsilon .
$$

Similarly as in the previous statement this can also be written as an improved version of (1), namely,

$$
\eta(K) \geq 1+\frac{2(n+1)}{n D} \delta\left(K, K^{*}\right)
$$

We also remark that it is not particularly important whether such results are stated in terms of the symmetric difference or the Hausdorff metric since there exist inequalities that allow the transition from one of these metrics to the other (see [4]).

Our second result concerns a theorem that was first published by Grünbaum [5]. In this article one also can find further references. As before let there be given a $K \in \mathcal{K}^{n}$ with centroid $z(K)$. Furthermore, let $E^{+}(u)$ denote the half-space whose boundary plane contains $z(K)$ and has $u$ as an interior normal vector. Define

$$
K_{u}=K \cap E^{+}(u)
$$

and

$$
\theta(K)=\sup \left\{v\left(K_{u}\right) / v\left(K_{-u}\right): u \in S^{n-1}\right\} .
$$

Similarly as $\eta(K)$ the function $\theta(K)$ can be conceived as a measure of symmetry. Obviously, $\theta(K) \geq 1$ and equality holds if $K$ is centrally symmetric. If $K$ is a cone and $u$ an outer normal vector of $K$ at its base, then

$$
\frac{v\left(K_{u}\right)}{v\left(K_{-u}\right)}=\left(1+\frac{1}{n}\right)^{n}-1 .
$$

The theorem just mentioned states that this is actually the extremal situation. In other words, if we define

$$
\theta_{n}=\left(1+\frac{1}{n}\right)^{n}-1,
$$

then

$$
\theta(K) \leq \theta_{n}
$$

with equality if $K$ is a cone. Our second theorem establishes a stability result for this inequality.

Theorem 2. Let there be given a $K \in \mathcal{K}^{n}$ and an $\varepsilon \geq 0$. There exists a constant $\lambda_{n}$, depending on $n$ only, such that the inequality $\theta(K) \geq \theta_{n}-\varepsilon$ implies that $K$ contains $a$ cone $C$ with the property that

$$
\Delta(K, C) \leq \lambda_{n} v(K) \varepsilon^{1 / 2 n^{2}} .
$$


Again, we add several pertinent remarks.

1. The proof will show that it would be possible to explicitly determine a suitable $\lambda_{n}$, but the additional technical details would require substantially more space.

2. Similarly as in the case of the first remark to Theorem 1 the above result can also be formulated as a sharpened version of (4), namely,

$$
\theta(K) \leq \theta_{n}-\left(\frac{1}{\lambda_{n} v(K)} \Delta(K, C)\right)^{2 n^{2}}
$$

and similarly as before one can state that $\theta(K)=\theta_{n}$ holds if and only if $K$ is a cone.

3. Although it is obvious that for a centrally symmetric $K$ we have $\theta(K)=1$, it is not obvious, but true, that this equality holds only in this case. For a detailed study of the equality $\theta(K)=1$, including pertinent stability results, see Section 5.6 of [3].

Proof of Theorem 1. If $\varepsilon \geq(n+1) / 4$, then (3) is trivially true for any inscribed cone $C$ in $K$. Consequently it can be assumed that

$$
\varepsilon<\frac{n+1}{4}
$$

Since $K$ is supposed to be given and fixed we frequently simplify the notation by writing $H$ and $h$ instead of $H_{K}$ and $h_{K}$, respectively. It suffices to consider only the case $\eta(K)=$ $n-\varepsilon$ since, if this is not so, it can be achieved by decreasing the value of $\varepsilon$. Then there exists a $u_{o} \in S^{n-1}$ such that $h\left(u_{o}\right) / h\left(-u_{o}\right)=n-\varepsilon$. Performing, if necessary, a suitable similarity transformation, we may assume that

$$
h\left(u_{o}\right)=n-\varepsilon, \quad h\left(-u_{o}\right)=1 .
$$

Let now $p$ be a point in $K \cap H\left(u_{o}\right)$, and let $E$ be the plane parallel to $H\left(u_{o}\right)$ and passing through $z(K)$. Obviously, there exists a cone $C$ with the following three properties: the apex of $C$ is $p$, its base is contained in $H\left(-u_{o}\right)$, and $E \cap C=E \cap K$. It will be shown that $C$ satisfies inequality (3). For this purpose we may suppose that the position of $K$ in $\mathbf{R}^{n}$ (equipped with the usual $\left(x_{1}, \ldots, x_{n}\right)$-coordinate system) is such that $u_{o}=(0, \ldots, 0,1)$ and

$$
z(C)=o .
$$

We write $E^{+}$and $E^{-}$for the two closed half-spaces determined by $E$, where $p \in E^{+}$. Furthermore, for any $X \in \mathcal{K}^{n}$ we let $X^{+}=X \cap E^{+}$and $X^{-}=X \cap E^{-}$. Note that $C^{+} \subset K^{+}, K^{-} \subset C^{-}$, and that

$$
\Delta(K, C)=\left(v\left(K^{+}\right)-v\left(C^{+}\right)\right)+\left(v\left(C^{-}\right)-v\left(K^{-}\right)\right) .
$$

Also, (7) shows that the height of $C$ is $n+1-\varepsilon$, and this implies that

$$
h_{C}\left(u_{o}\right)=n\left(1-\frac{\varepsilon}{n+1}\right), \quad h_{C}\left(-u_{o}\right)=1-\frac{\varepsilon}{n+1} .
$$

For any $Y \in \mathcal{K}^{n}$ let $z_{n}(Y)$ denote the $x_{n}$-coordinate of $z(Y)$. Since $z_{n}(K)=h_{C}\left(u_{o}\right)-$ $h_{K}\left(u_{o}\right)$ it follows from (7) and (10) that $z_{n}(K)=\varepsilon /(n+1)$. Thus we can state that

$$
\frac{1}{v(K)}\left(v\left(K^{+}\right) z_{n}\left(K^{+}\right)+v\left(K^{-}\right) z_{n}\left(K^{-}\right)\right)=\frac{\varepsilon}{n+1}
$$


and, as a consequence of (8),

$$
v\left(C^{+}\right) z_{n}\left(C^{+}\right)+v\left(C^{-}\right) z_{n}\left(C^{-}\right)=0 .
$$

Hence,

$$
\left(v\left(K^{+}\right) z_{n}\left(K^{+}\right)-v\left(C^{+}\right) z_{n}\left(C^{+}\right)\right)+\left(v\left(K^{-}\right) z_{n}\left(K^{-}\right)-v\left(C^{-}\right) z_{n}\left(C^{-}\right)\right)=\frac{\varepsilon}{n+1} v(K) .
$$

We also note that (2) (applied to $K^{+}$) and the fact that $\eta\left(C^{+}\right)=n$ imply that

$$
z_{n}\left(K^{+}\right) \geq z_{n}\left(C^{+}\right)
$$

We now proceed under the hypothesis that in addition to (12) we have

$$
z_{n}\left(K^{-}\right) \geq z_{n}\left(C^{-}\right)
$$

From (11), (12), (13), and the fact that $z_{n}\left(C^{-}\right) \leq 0$ we infer that

$$
\left(v\left(K^{+}\right)-v\left(C^{+}\right)\right) z_{n}\left(C^{+}\right)+\left(v\left(C^{-}\right)-v\left(K^{-}\right)\right)\left|z_{n}\left(C^{-}\right)\right| \leq \frac{\varepsilon}{n+1} v(K) .
$$

Using (10) we see that $z_{n}\left(C^{+}\right)=(1 /(n+1)) h_{C}\left(u_{o}\right) \geq(n-\varepsilon) /(n+1)$, and it follows from (6) that

$$
z_{n}\left(C^{+}\right) \geq \frac{1}{4} .
$$

Furthermore, since the height of the truncated cone $C^{-}$equals $h\left(-u_{o}\right)=1$ the orthogonal distance of $z\left(C^{-}\right)$from the base of $C$ is at most $\frac{1}{2}$. This implies that $\left|z_{n}\left(C^{-}\right)\right| \geq$ $h_{C}\left(-u_{o}\right)-\frac{1}{2}$. Consequently, using again (6) and (10), we obtain

$$
\left|z_{n}\left(C^{-}\right)\right| \geq \frac{1}{2}-\frac{\varepsilon}{n+1} \geq \frac{1}{4} .
$$

Clearly, (14), (15), and (16) imply

$$
\left(v\left(K^{+}\right)-v\left(C^{+}\right)\right)+\left(v\left(C^{-}\right)-v\left(K^{-}\right)\right) \leq \frac{4 \varepsilon}{n+1} v(K) .
$$

Inequality (3) is now an obvious consequence of this inequality and (9).

To complete the proof we need to show (13). First we note that it can be assumed that $C^{-}$and $K^{-}$have rotational symmetry with respect to the $x_{n}$-axis. This assumption is justified since one can perform a rotational symmetrization ("Schwarzsche Abrundung," see Section 41 of [1]) with respect to the $x_{n}$-axis, and it is obvious that this symmetrization does not change $z_{n}\left(K^{-}\right)$and $z_{n}\left(C^{-}\right)$. Thus we can proceed under the assumption that $C^{-}$ is a truncated cone whose "upper base" $B^{*}=C \cap E$ and "lower base" $B_{*}=C \cap H\left(-u_{o}\right)$ are $(n-1)$-dimensional balls. Let now $E^{\prime}$ be the plane that is parallel to $E$ and contains $z\left(K^{-}\right)$, and let $K^{\prime}$ be the truncated cone (possibly cylinder) with upper base $B^{*}$, lower base contained in $B_{*}$, and such that $K^{-} \cap E^{\prime}=K^{\prime} \cap E^{\prime}$. Since it is evident that $z_{n}\left(K^{\prime}\right) \leq z_{n}\left(K^{-}\right)$we only have to show that

$$
z_{n}\left(K^{\prime}\right) \geq z_{n}\left(C^{-}\right)
$$


For this purpose let $r, R$, and $\rho$ denote, respectively, the radii of $B^{*}, B_{*}$, and that of the lower base of $K^{\prime}$, and let $h$ signify the height of $C^{-}$. If $\rho \leq r$, then obviously $z_{n}\left(K^{\prime}\right) \geq$ $h_{C}\left(-u_{o}\right)+h / 2$ and $z_{n}\left(C^{-}\right) \leq h_{C}\left(-u_{o}\right)+h / 2$, and consequently $z_{n}\left(K^{\prime}\right) \geq z_{n}\left(C^{-}\right)$. If $\rho>r$ let $\alpha=R / r$ and $\beta=\rho / r$. Then $\alpha \geq \beta>1$, and an elementary calculation shows that

$$
z_{n}\left(K^{\prime}\right)-z_{n}\left(C^{-}\right)=\frac{h}{n+1} \sum_{k=1}^{n-1}\left(\frac{\beta^{k}-1}{\beta^{n}-1}-\frac{\alpha^{k}-1}{\alpha^{n}-1}\right) .
$$

Since it is easily checked that for $1 \leq k \leq n-1$ and $x>1$ the functions $\left(x^{k}-\right.$ $1) /\left(x^{n}-1\right)$ are decreasing we obtain the desired inequality. This concludes the proof of Theorem 1 .

The following lemma concerning the symmetric difference metric will facilitate the proof of Theorem 2. It is also of some independent interest. In the proof of this lemma and in the proof of Theorem 2 we let $c_{1}, \ldots, c_{14}$ denote positive constants that depend on $n$ only. The symbol $\|\cdot\|$ denotes the euclidean norm in $\mathbf{R}^{n}$.

Lemma. Let $M, N \in \mathcal{K}^{n}$, and assume that $v(M \cap N)>0$ and $v(M) \leq 1, v(N) \leq 1$. Then there exists a homothetic copy $N^{\prime}$ of $N$ such that $N^{\prime} \subset N \cap M$ and

$$
\Delta\left(M, N^{\prime}\right) \leq \gamma_{n}\left(\frac{\Delta(M, N)}{v(M \cap N)}\right)^{1 / n},
$$

where $\gamma_{n}$ depends on $n$ only.

Proof. Let $\mathcal{L}$ denote the Loewner ellipsoid of $M \cap N$, that is, the ellipsoid of smallest volume that contains $M \cap N$. Since a volume preserving affine transformation of $\mathbf{R}^{n}$ leaves all entities in (17) invariant it can be assumed that $\mathcal{L}$ is a ball centered at $o$. Let $r$ denote the radius of $\mathcal{L}$. Then the well-known theorem of John states that $(1 / n) \mathcal{L} \subset M \cap N$, and this shows that

$$
\left(\frac{1}{n}\right)^{n} v(\mathcal{L}) \leq v(M \cap N) \leq v(\mathcal{L}) .
$$

Now, if $x \in N \backslash M$ let $x^{\prime}$ be the intersection point of the line segment $[o, x]$ with the boundary of $M$. There obviously are an $x_{o} \in N \backslash M$ and a corresponding $x_{o}^{\prime}$ such that $\left\|x_{o}^{\prime}\right\| /\left\|x_{o}\right\|=\inf \left\{\left\|x^{\prime}\right\| /\|x\|: x \in N \backslash M\right\}$. Hence, for all $x \in N \backslash M$ we have

$$
\frac{\left\|x_{o}^{\prime}\right\|}{\left\|x_{o}\right\|}\|x\| \leq\left\|x^{\prime}\right\|
$$

and this implies that

$$
\frac{\left\|x_{o}^{\prime}\right\|}{\left\|x_{o}\right\|} N \subset M
$$

Let $P$ be a support plane of $M$ at $x_{o}^{\prime}$ and consider the cones $U=\mathcal{C}\left(x_{o},(1 / n) \mathcal{L} \cap\left(P-x_{o}^{\prime}\right)\right)$ and $U^{\prime}=\mathcal{C}\left(x_{o}, U \cap P\right)$. Observing that the base of $U$ is an $(n-1)$-dimensional ball of 
radius $(1 / n) r$ and the height of $U$ is at least $(1 / n) r$ we find

$$
\Delta(M, N) \geq v\left(U^{\prime}\right) \geq\left(\frac{\left\|x_{o}\right\|-\left\|x_{o}^{\prime}\right\|}{\left\|x_{o}\right\|}\right)^{n} v(U) \geq c_{1}\left(1-\frac{\left\|x_{o}^{\prime}\right\|}{\left\|x_{o}\right\|}\right)^{n} v(\mathcal{L}) .
$$

Combined with (18) this yields

$$
1-\left(c_{2} \frac{\Delta(M, N)}{v(M \cap N)}\right)^{1 / n} \leq \frac{\left\|x_{o}^{\prime}\right\|}{\left\|x_{o}\right\|} .
$$

Now, if

$$
c_{2} \frac{\Delta(M, N)}{v(M \cap N}<1
$$

let

$$
N^{\prime}=\left(1-\left(c_{2} \frac{\Delta(M, N)}{v(M \cap N}\right)^{1 / n}\right) N .
$$

Then (19) shows that $N^{\prime} \subset M \cap N$ and one deduces that

$$
\begin{aligned}
\Delta\left(M, N^{\prime}\right) & \leq \Delta(M, N)+\Delta\left(N, N^{\prime}\right) \\
& \leq \Delta(M, N)+\left(1-\left(1-\left(c_{2} \frac{\Delta(M, N)}{v(M \cap N}\right)^{1 / n}\right)^{n}\right) v(N) .
\end{aligned}
$$

Using also the inequalities $v(N) \leq 1, v(M \cap N) \leq 1$, and $\Delta(M, N) \leq v(M)+v(N) \leq 2$ we readily deduce that

$$
\Delta\left(M, N^{\prime}\right) \leq c_{3}\left(\frac{\Delta(M, N)}{v(M \cap N)}\right)^{1 / n} .
$$

If (20) is not satisfied then, taking as $N^{\prime}$ any homothetic copy of $N$ contained in $M \cap N$, one obtains

$$
\Delta\left(M, N^{\prime}\right) \leq v(M) \leq 1 \leq\left(c_{2} \frac{\Delta(M, N)}{v(M \cap N)}\right)^{1 / n} .
$$

Thus, letting $\gamma_{n}=\max \left\{c_{3}, c_{2}^{1 / n}\right\}$, we have proved (17).

Proof of Theorem 2. One obviously may assume that

$$
v(K)=1 .
$$

Also, replacing, if necessary, $\lambda_{n}$ by $\max \left\{\lambda_{n}, 1\right\}$ we see that (5) is trivially satisfied if $\varepsilon \geq 1$. Thus it can be assumed that

$$
\varepsilon<1 \text {. }
$$

Moreover, there is no loss in generality by assuming that

$$
z(K)=o
$$


and that $\theta(K)=\theta_{n}-\varepsilon=v\left(K_{u_{o}}\right) / v\left(K_{-u_{o}}\right)$, where, in the given $\left(x_{1}, \ldots, x_{n}\right)$-coordinate system, $u_{o}=(0, \ldots, 0,1)$. Then $v\left(K_{u_{o}}\right)=\left(\theta_{n}-\varepsilon\right) /\left(\theta_{n}-\varepsilon+1\right)$, and observing (22) we deduce that

$$
\frac{1}{9} \leq 1-\left(\frac{n}{n+1}\right)^{n}-\frac{4}{9} \varepsilon \leq v\left(K_{u_{o}}\right) \leq 1-\left(\frac{n}{n+1}\right)^{n} .
$$

The plane $x_{n}=0$ will be denoted by $F$, and, similarly as before, we write $F^{+}$and $F^{-}$ for the two closed half-spaces defined by $x_{n} \geq 0$ and $x_{n} \leq 0$, respectively. Furthermore, for any $Y \in \mathcal{K}^{n}$ we again set $Y^{+}=Y \cap F^{+}, Y^{-}=Y \cap F^{-}$. Thus, $K_{u_{o}}=K^{+}$and $K_{-u_{o}}=K^{-}$. The $x_{n}$-axis will be denoted by $\ell$.

We first deal with the case when $K$ is rotationally symmetric with respect to $\ell$ and prove under this assumption the following statement:

Let $C_{o}$ be the cone in $\mathbf{R}^{n}$ with the following properties: The base of $C_{o}$ is an $(n-1)$ dimensional ball in $F^{+}$centered at $\ell$ and parallel to $F$, and the apex of $C_{o}$ is in $\ell \cap F^{-}$. Moreover, $K \cap F=C_{o} \cap F$, and $v\left(C_{o}^{+}\right)=v\left(K^{+}\right), v\left(C_{o}^{-}\right)=v\left(K^{-}\right)$. Then

$$
\Delta\left(K, C_{o}\right) \leq c_{4} \varepsilon^{1 / 2 n} .
$$

It is evident that a cone with the above properties exists. The idea to construct this cone is suggested by the work of Grünbaum [5] where it is used to prove (4). Note that (21) implies

$$
v\left(C_{o}\right)=1 .
$$

To prove (24) we first show that $v\left(K \cap C_{o}\right)$ is not less than some constant (depending on $n$ only). Let $Q$ be the cone with apex $q$ at $H_{K}\left(-u_{o}\right) \cap \ell$, base in $H_{K}\left(u_{o}\right)$, and such that $K \cap F=Q \cap F$. Clearly, $K^{+} \subset Q$ and it follows from (23) that

$$
v(Q) \geq v\left(K^{+}\right) \geq \frac{1}{9} .
$$

Letting $Q^{\prime}=\mathcal{C}(q, K \cap F)$ we see that $Q^{\prime} \subset K \cap C_{o}$ and therefore

$$
v\left(K \cap C_{o}\right) \geq v\left(Q^{\prime}\right)=\left(\frac{h_{K}\left(-u_{o}\right)}{h_{K}\left(u_{o}\right)+h_{K}\left(-u_{o}\right)}\right)^{n} v(Q) .
$$

Combining this with (26) and the fact that, due to (2), $h_{K}\left(-u_{o}\right) /\left(h_{K}\left(u_{o}\right)+h_{K}\left(-u_{o}\right)\right) \geq$ $1 /(n+1)$ we obtain the desired inequality

$$
v\left(K \cap C_{o}\right) \geq c_{5}
$$

Next, consider the Loewner ellipsoid, say $\mathcal{E}$, of $K \cap C_{o}$. From the uniqueness of $\mathcal{E}$ and the axial symmetry of $K$ and $C_{o}$ it follows immediately that $\mathcal{E}$ also has $\ell$ as an axis of symmetry. Consequently there exists a volume preserving affine transformation $\sigma$ of the form $\left(x_{1}, \ldots, x_{n-1}, x_{n}\right) \rightarrow\left(\lambda x_{1}, \ldots, \lambda x_{n-1}, \lambda^{1-n} x_{n}\right)(\lambda>0)$ that transforms $\mathcal{E}$ into a ball $\sigma \mathcal{E}$. Since (24) is invariant with respect to $\sigma$ it suffices to prove this inequality under the assumption that $\mathcal{E}$ is already a ball. Then it follows from John's theorem and (27) that $K \cap C_{o}$ contains a ball $B$ such that

$$
v(B) \geq c_{6} .
$$


Because of (21) and (25) this implies in particular that the diameters of both $K$ and $C_{o}$ are bounded by constants depending on $n$ only.

It is now convenient to introduce the following notation:

$$
K_{1}=K^{+} \backslash C_{o}^{+}, \quad K_{2}=K^{-} \backslash C_{o}^{-}, \quad C_{1}=C_{o}^{+} \backslash K^{+}, \quad C_{2}=C_{o}^{-} \backslash K^{-} .
$$

The definition of $C_{o}$ shows that

$$
v\left(K^{+}\right)=v\left(C^{+}\right), \quad v\left(K^{-}\right)=v\left(C^{-}\right), \quad v\left(K_{1}\right)=v\left(C_{1}\right), \quad v\left(K_{2}\right)=v\left(C_{2}\right),
$$

and consequently

$$
\Delta\left(K, C_{o}\right)=2\left(v\left(K_{1}\right)+v\left(K_{2}\right)\right)
$$

Letting again $z_{n}(X)$ denote the $x_{n}$-coordinate of $z(X)$, and observing that $K=(K \cap$ $\left.C_{o}\right) \cup K_{1} \cup K_{2}, C_{o}=\left(K \cap C_{o}\right) \cup C_{1} \cup C_{2}$, where the unions are disjoint, we have

$$
\begin{array}{r}
v\left(K \cap C_{o}\right) z_{n}\left(K \cap C_{o}\right)+v\left(K_{1}\right) z_{n}\left(K_{1}\right)+v\left(K_{2}\right) z_{n}\left(K_{2}\right)=z_{n}(K)=0, \\
v\left(K \cap C_{o}\right) z_{n}\left(K \cap C_{o}\right)+v\left(C_{1}\right) z_{n}\left(C_{1}\right)+v\left(C_{2}\right) z_{n}\left(C_{2}\right)=z_{n}\left(C_{o}\right) .
\end{array}
$$

Since $z_{n}\left(C_{o}\right)>0$ would imply $v\left(K^{+}\right)=v\left(C_{o}^{+}\right)>1-(n /(n+1))^{n}$, which contradicts (23), we see that $z_{n}\left(C_{o}\right) \leq 0$. This fact in conjunction with (29), (31), and (32) shows that

$$
\left|z_{n}\left(C_{o}\right)\right|=v\left(K_{1}\right)\left(z_{n}\left(K_{1}\right)-z_{n}\left(C_{1}\right)\right)+v\left(K_{2}\right)\left(z_{n}\left(K_{2}\right)-z_{n}\left(C_{2}\right)\right) .
$$

We now wish to establish a lower bound for $z_{n}\left(K_{1}\right)-z_{n}\left(C_{1}\right)$. If $s$ denotes the distance between $F$ and the base of $C_{o}$, then obviously

$$
z_{n}\left(C_{1}\right) \leq s
$$

On the other hand, if $t$ is the distance between the support plane $H_{K}\left(u_{o}\right)$ and the base of $C_{o}$, then (because of (2))

$$
z_{n}\left(K_{1}\right)-s \geq \frac{1}{n+1} t
$$

Hence,

$$
z_{n}\left(K_{1}\right)-z_{n}\left(C_{1}\right) \geq \frac{1}{n+1} t .
$$

Since $K_{1}$ is outside $C_{o}$, but within the conical extension of $C_{o}$ up to $H_{K}\left(u_{o}\right)$ it follows that

$$
\left(\frac{h+t}{h}\right)^{n}-1 \geq v\left(K_{1}\right),
$$

where $h$ signifies the height of $C_{o}$. Observing that $t \leq c_{7}$ and, because of (28), $h \geq c_{8}$ we deduce that

$$
t \geq c_{9} v\left(K_{1}\right)
$$


In conjunction with (34) this yields

$$
z_{n}\left(K_{1}\right)-z_{n}\left(C_{1}\right) \geq c_{10} v\left(K_{1}\right) .
$$

Next, we derive a lower bound for $z_{n}\left(K_{2}\right)-z_{n}\left(C_{2}\right)$. If $q=\left(0, \ldots, 0, q_{n}\right)$ denotes again the point $H\left(-u_{o}\right) \cap \ell$, then $K^{-} \cap C_{o}^{-}$contains the cone $\mathcal{C}(q, K \cap F)$. Consequently, if $g$ denotes the height of $C_{o}^{-}$and if $v^{\prime}$ indicates the volume for $(n-1)$ dimensional convex bodies, then

$$
\begin{aligned}
\frac{1}{n} v^{\prime}(K \cap F)\left|q_{n}\right| & \leq v\left(K^{-} \cap C_{o}^{-}\right)=v\left(K^{-}\right)-v\left(K_{2}\right)=v\left(C_{o}^{-}\right)-v\left(K_{2}\right) \\
& =\frac{1}{n} v^{\prime}(K \cap F) g-v\left(K_{2}\right) .
\end{aligned}
$$

Recalling the remark after (28) regarding the boundedness of $K$ we obtain

$$
g-\left|q_{n}\right| \geq \frac{n v\left(K_{2}\right)}{v^{\prime}(K \cap F)} \geq c_{11} v\left(K_{2}\right) .
$$

Thus, if $p$ denotes the apex of $C_{o}$, the cone $\mathcal{C}\left(p, C_{o} \cap H_{K}\left(-u_{o}\right)\right)$ has height at least $c_{11} v\left(K_{2}\right)$.

Let now $F^{\prime}$ be the plane that separates $K_{2}$ and $C_{2}$, and set $C_{3}=\mathcal{C}\left(p, C_{o} \cap F^{\prime}\right)$. Obviously,

$$
\mathcal{C}\left(p, C_{o} \cap H_{K}\left(-u_{o}\right)\right) \subset C_{3},
$$

and if $h_{3}$ denotes the height of $C_{3}$ one obtains from (36) that

$$
h_{3} \geq c_{11} v\left(K_{2}\right) \text {. }
$$

Furthermore, we can show that

$$
z_{n}\left(C_{2}\right) \leq z_{n}\left(C_{3}\right)
$$

To verify this let $K_{3}=C_{3} \cap K$. Then $C_{3}=C_{2} \cup K_{3}$, and since $C_{2}$ and $K_{3}$ are disjoint it follows that $\left(z_{n}\left(C_{3}\right)-z_{n}\left(C_{2}\right)\right) v\left(C_{2}\right)=\left(z_{n}\left(K_{3}\right)-z_{n}\left(C_{3}\right)\right) v\left(K_{3}\right)$. Thus one only has to show that $z_{n}\left(C_{3}\right) \leq z_{n}\left(K_{3}\right)$. However, this can easily be seen by constructing the cone $C_{4}$ which has the same base as $C_{3}$ and is such that $C_{4} \cap F^{\prime \prime}=K_{3} \cap F^{\prime \prime}$, where $F^{\prime \prime}$ is the plane that passes through $z\left(K_{3}\right)$ and is parallel to $F$. Evidently $z_{n}\left(C_{3}\right) \leq z_{n}\left(C_{4}\right) \leq z_{n}\left(K_{3}\right)$.

From (37), (38), (39), and the fact that $z_{n}\left(K_{2}\right)-z_{n}\left(C_{3}\right)$ is not less than the distance of $z\left(C_{3}\right)$ from $F^{\prime}$ (since $z\left(K_{2}\right)$ and $z\left(C_{3}\right)$ lie on different sides of $F^{\prime}$ ) one can deduce that

$$
z_{n}\left(K_{2}\right)-z_{n}\left(C_{2}\right) \geq z_{n}\left(K_{2}\right)-z_{n}\left(C_{3}\right) \geq \frac{1}{n+1} h_{3} \geq c_{12} v\left(K_{2}\right) .
$$

We can now complete the proof of (24). From (33), (35), and (40) one deduces that

$$
\left|z_{n}\left(C_{o}\right)\right| \geq c_{10} v\left(K_{1}\right)^{2}+c_{12} v\left(K_{2}\right)^{2} .
$$

Noting that $v\left(K_{1}\right)^{2}+v\left(K_{2}\right)^{2} \geq \frac{1}{2}\left(v\left(K_{1}\right)+v\left(K_{2}\right)\right)^{2}$ we infer, observing also (30), that

$$
\left|z_{n}\left(C_{o}\right)\right| \geq c_{13} \Delta\left(K, C_{o}\right)^{2} .
$$


Since the height of $C_{o}^{-}$is $(n /(n+1)) h+\left|z_{n}\left(C_{o}\right)\right|$ and, as noted before, $h \geq c_{8}$ it follows from this inequality together with (21), (25), and (29) that

$$
1-v\left(K^{+}\right)=v\left(C_{o}^{-}\right)=\frac{1}{h^{n}}\left(\frac{n}{n+1} h+\left|z_{n}\left(C_{o}\right)\right|\right)^{n} \geq\left(\frac{n}{n+1}+c_{14} \Delta\left(K, C_{o}\right)^{2}\right)^{n} .
$$

Combining this with (23) we obtain

$\frac{n}{n+1}+\left(\frac{4}{9} \varepsilon\right)^{1 / n} \geq\left(\left(\frac{n}{n+1}\right)^{n}+\frac{4}{9} \varepsilon\right)^{1 / n} \geq\left(1-v\left(K^{+}\right)\right)^{1 / n} \geq \frac{n}{n+1}+c_{14} \Delta\left(K, C_{o}\right)^{2}$ and this implies (24).

Applying the above lemma with $M=K$ and $N=C_{o}$, and taking into account (21), (24), (25), and (27), we see that $K$ contains a cone $C^{\prime}$ that is homothetic to $C_{o}$ and satisfies the inequality

$$
v(K)-v\left(C^{\prime}\right)=\Delta\left(K, C^{\prime}\right) \leq \lambda_{n} \varepsilon^{1 / 2 n^{2}},
$$

where $\lambda_{n}$ depends on $n$ only.

Finally, if $K$ lacks the stipulated axial symmetry we perform a rotational symmetrization of $K$ with respect to the line $\ell$. This transforms $K$ into a convex body $\tilde{K}$ that has $\ell$ as an axis of symmetry and has the property that $v(\tilde{K})=v(K)=1$. Then (41) applied to $\tilde{K}$ instead of $K$ shows that $\tilde{K}$ contains a cone $\tilde{C}$ that has $\ell$ as an axis of symmetry, and whose base, say $\tilde{J}$, is in a plane $\tilde{F}$, that is orthogonal to $\ell$. Moreover, we have

$$
v(\tilde{K})-v(\tilde{C}) \leq \lambda_{n} \varepsilon^{1 / 2 n^{2}} .
$$

Let now $C$ be defined as a cone with base $K \cap \tilde{F}$ and apex in $K \cap H_{K}\left(-u_{o}\right)$. Since the spherical symmetrization has the property that $v^{\prime}(\tilde{K} \cap \tilde{F})=v^{\prime}(K \cap \tilde{F})$ and since $\tilde{J} \subset \tilde{K} \cap \tilde{F}$ it follows that the $(n-1)$-dimensional volume of the base of $C$ is at least that of the base of $\tilde{C}$ and the height of $C$ is at least that of $\tilde{C}$. Hence,

$$
\Delta(K, C)=v(K)-v(C) \leq v(\tilde{K})-v(\tilde{C}) \leq \lambda_{n} \varepsilon^{1 / 2 n^{2}},
$$

and this proves Theorem 2 .

\section{References}

1. T. Bonnesen and W. Fenchel, Theorie der konvexen Körper. Ergebn. d. Math., Bd. 3, Springer-Verlag, Berlin, 1934. (English translation: Theory of Convex Bodies, BCS, Moscow, ID, 1987.)

2. H. Groemer, Stability of geometric inequalities. In Handbook of Convex Geometry (P. M. Gruber and J. M. Wills, Editors), Section 1.4. North-Holland, Amsterdam, 1993.

3. H. Groemer, Geometric Applications of Fourier Series and Spherical Harmonics. Cambridge University Press, Cambridge, 1996.

4. H. Groemer, On the symmetric difference metric for convex bodies. To appear in Beiträge Algebra Geom.

5. B. Grünbaum, Partitions of mass distributions and of convex bodies by hyperplanes. Pacific J. Math 10 (1960), 1257-1261.

6. B. Grünbaum, Measures of symmetry of convex sets. In Proceedings of Symposia in Pure Mathematics, Vol. VII (V. L. Klee, Editor), pp. 233-270. American Mathematical Society, Providence, RI, 1963.

Received January 4, 1999, and in revised form May 24, 1999. Online publication May 8, 2000. 